\title{
Radiologic intervention due to delayed gastric emptying after pylorus preserving gastrectomy for gastric cancer does not affect pyloric function
}

Hyun Tae Lim', Shin-Hoo Park', Jong-Ho Choi', Jae Seok Bae², Seong-Ho Kong'1, Do Joong Park', Hyuk-Joon Lee', Se Hyung Kim², Han-Kwang Yang ${ }^{1}$

Departments of 'Surgery and 'Radiology, Seoul National University College of Medicine, Seoul, Korea

Purpose: The purpose of this study is to evaluate whether radiologic intervention in the pylorus decreases its function for delayed gastric emptying (DGE) patients after pylorus preserving gastrectomy (PPG) for gastric cancers and to determine the optimal interventional algorithm.

Methods: PPG patients who underwent intervention for DGE from January 2013 to December 2017 and a control group using propensity score matching were identified. Pyloric function was compared by subjective symptoms, postoperative upper gastrointestinal series at 3 months (short-term function), and esophagogastroduodenoscopy findings at 12 months (long-term function). Serum albumin levels and body weight change, 6 months and 12 months postoperatively, were compared to evaluate nutritional status. Interventional success rate, mean hospital stay, and recurrence of DGE were analyzed to determine the optimal intervention plan.

Results: Fifty-one out of 677 patients (7.53\%) received intervention. There was no difference in pyloric function and nutritional status between the intervention and control groups. The interventional success rate for first-time balloon dilatation was 41.7\% (20/48). If a second intervention was required and balloon dilatation was done, the success rate was $45.5 \%(5 / 11)$. However, if stent insertion was done, the success rate was 100\% (17/17). Subsequent stent insertion after balloon dilatation resulted in a shorter mean hospital stay. Intervention including stent insertion had a lower recurrence of DGE than balloon only intervention (1.96\% vs. 5.88\%, $P=0.041$ ).

Conclusion: Radiologic intervention did not decrease long-term pyloric function. For treating DGE, if at first balloon dilatation fails, retrievable stent insertion can be considered as a second choice.

Keywords: Stomach neoplasms, Pylorus-presening gastrectomy, Gastroparesis, Treatment outcome

Received: Sep 29, 2020 Revised: Nov 8, 2020 Accepted: Nov 18, 2020

Correspondence to: Han-Kwang Yang

Department of Surgery, Seoul National University Hospital and Cancer

Research Institute, Seoul National University College of Medicine,

101 Daehak-ro, Jongno-gu, Seoul 03080, Korea

Tel: +82-2-2072-2817, Fax: +82-2-766-3975

E-mail: hkyang@snu.ac.kr

ORCID: Hyun Tae Lim (https://orcid.org/0000-0001-6934-5013), Shin-Hoo Park (https://orcid.org/0000-0001-9767-6100), Jong-Ho Choi (https://orcid.org/00000001-6963-7075), Jae Seok Bae (https://orcid.org/0000-0003-2768-7917), SeongHo Kong (https://orcid.org/0000-0002-3929-796X), Do Joong Park (https://orcid. org/0000-0001-9644-6127), Hyuk-Joon Lee (https://orcid.org/0000-0001-76242020), Se Hyung Kim (https://orcid.org/0000-0001-8664-0356), Han-Kwang Yang (https://orcid.org/0000-0003-3495-3048)

\section{Copyright (C) 2020 Korean Society of Surgical Oncology}

This is an Open Access article distributed under the terms of the Creative Commons Attribution Non-Commercial License (http://creativecommons.org/licenses/by-nc/4.0) which permits unrestricted non-commercial use, distribution, and reproduction in any medium, provided the original work is properly cited.

\section{INTRODUCTION}

Pylorus preserving gastrectomy (PPG) is a surgical technique used for early gastric cancer located in the middle portion of the stomach with the distal tumor border being at least $5 \mathrm{~cm}$ proximal to the pylorus [1,2]. Studies have shown that compared to distal gastrectomy (DG), PPG has lower incidence of dumping syndrome, bile reflux, gallstone formation, and improved nutritional status with comparable oncologic outcomes [3-12]. However, one of the most concerning complications is delayed gastric emptying (DGE) [4,13-20]. Radiologic intervention is generally used in such cases and the method used in our center includes balloon dilatation and retrievable stent insertion [21-23]. Radiologic studies from our center showed that balloon dilation is a safe and effective treatment for patients with pyloric spasms after PPG and for patients who were refractory to balloon dilations, retrievable stent placement 
can be a safe alternative tool [24]. In that study, authors conducted telephone interviews of patients who underwent such interventions and efficacy of the intervention was determined by the patients' subjective symptoms. However, post-interventional outcomes in terms of pyloric function and the specific treatment algorithm of DGE patients has not been described to date.

The purpose of this study is to evaluate if mechanical intervention of the pylorus decreases its function when treating DGE patients after PPG, and to devise the most optimal treatment algorithm for such patients. Our hypothesis was that radiologic intervention does not decrease pyloric function.

\section{METHODS}

PPG patients were identified in the prospectively collected clinical gastric cancer database at Seoul National University Hospital from January 2013 to December 2017, a total period of 5 years. The data from this database, including complications of inpatients, has been collected and updated at a weekly-based conference. Propensity score matching, adjusting for sex, age and type of surgery was used to obtain a non-intervention control group of two times the intervention group.

DGE was suspected in patients with symptoms of gastroparesis such as nausea or bloating with simple abdomen X-ray showing gastric distension.

Demographic data, which included sex, age, type of surgery, tumor location and distance from pylorus was obtained. Clinicopathologic characteristics using the Japanese classification of gastric cancer and the eighth edition of the American Joint Committee on Cancer TNM classification.

Radiologic interventionists carried out both balloon dilatation and retrievable stent insertion. For balloon dilatation, a 4-6 cm long, 16-25 $\mathrm{mm}$ balloon was placed over the stenotic pyloric canal. For stent placement, an 8-15 cm long, $20 \mathrm{~mm}$ diameter fully covered stent was placed across the stenotic pyloric canal. One end of a string was attached to the proximal edge of the stent and the other end was passed through the stomach and esophagus, exiting the nostril and anchored around the ear. The string prevented distal migration of the stent and facilitated stent removal. The stent was retained for 1-2 weeks. However, if migration of stent was confirmed by simple abdomen $\mathrm{X}$-ray, the stent was removed earlier. After each procedure, the presence of complications such as extraluminal leakage was checked via contrast injection and fluoroscopy.

Both interventions required the patient to maintain nil per os from the day of intervention. Sips of water were started from the next day, followed by semi fluid diet and soft bland diet at 1-day intervals. The decision to move on to the next diet plan was made if the patient did not complain of abdominal distension and abdominal X-ray showed no gastric distension.

Patients who underwent radiologic intervention for DGE were subcategorized into two different groups, patients who received balloon dilatation only (balloon only group) and patients who received retrievable stent insertion as a primary intervention or after trial of balloon dilatation (stent group).

Pyloric function was compared by three specific variables of subjective symptoms, postoperative upper gastrointestinal series (UGIS) at 3 months and esophagogastroduodenoscopy (EGD) findings at 12 months. All postoperative patients were asked about subjective symptoms related to dumping syndrome and gastric stasis. Symptoms suggesting dumping syndrome implied a decrease in pyloric function and consisted of symptoms such as reflux, diarrhea, nausea, dizziness, etc. Symptoms suggesting gastric stasis consisted of bloating, epigastric fullness, etc. If the patient complained of epigastric fullness and simple abdomen X-ray showed signs of gastric distension, admission and work-up including UGIS was done to determine if there was a recurrence of DGE. Routine outpatient commitment (OPC) follow-up was done twice (once every 2 weeks) after discharge. After the two 2-week intervals, the patient was followed up at 3 months, then 6 months later. UGIS was done at 3 months and EGD was done at 12 months postoperatively. Both UGIS and EGD findings were categorized into four different subgroups depending on their findings: "no specific finding," "remnant food," "signs of gastroesophageal reflux" (e.g., contrast reflux for UGIS and mucosal break, ulceration for EGD), and "pylorus abnormality" (e.g., pyloric spasm for UGIS and narrowing for EGD). Findings showing "remnant food" or "signs of gastroesophageal reflux," may be due to a decrease in pyloric function. Findings showing "remnant food" may be due to pyloric stenosis. Therefore, "pylorus abnormality" was also identified to rule out such findings. Due to the timing of such studies, UGIS findings were used to determine short-term pyloric function and EGD findings were used to determine long-term pyloric function.

Nutritional status including serum albumin levels and body weight change was determined, 6 months and 12 months postoperatively. The hospital stay from our study was defined as the time from the date of first intervention until the discharge day. If a patient required more than one intervention, that patient's hospital stay was cumulatively added and the overall mean hospital stay was calculated.

The interventional success rate was defined as the rate in which the patient was successfully discharged after that particular intervention. The recurrence of DGE, in our study, was defined as read- 
mission for further intervention after successful post-interventional discharge.

Independent t-test was used to compare between the control group and the intervention group for demographics, between intervention and non-intervention group for postoperative pyloric function and nutritional status, and between the balloon intervention and stent insertion group for recurrence of DGE. All tests were performed at a significance level of 5\% using SPSS version 19.0 (SPSS, Inc., Chicago, IL, USA).

The same standardized surgical technique, which included the preservation of the infrapyloric artery and the preservation of the hepatic branch of the vagus nerve was used for all surgeons in our institution for PPG. The study protocol was approved by the Institutional Review Board of Seoul National University Hospital (IRB No. H-2003-196-1112). All procedures followed were in accordance with the ethical standards of the responsible committee on human experimentation (institutional and national) and with the Helsinki Declaration of 1964 and later versions. Informed consent or a substitute for it was obtained from all patients for being included in the study.

\section{RESULTS}

\section{Patients}

A total of 677 patients underwent PPG during the study period and 51 patients who underwent intervention (7.53\%) were identified, and the control group of 102 patients was obtained by propensity score matching. Out of the 51 patients, there were 25 patients

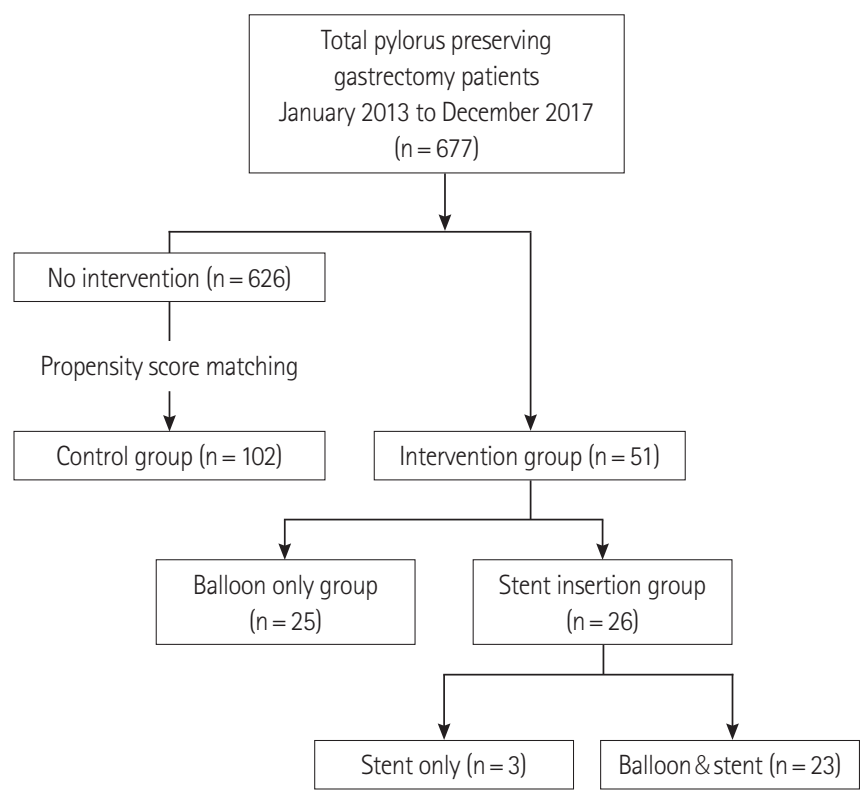

Fig. 1. Study patients.
$(49.0 \%)$ in the balloon only group. In the stent group, 23 patients (45.1\%) received balloon and stent intervention and the remaining three patients (5.9\%) received stent only intervention (Fig. 1).

When comparison was made between the intervention and control groups, there was no significant difference in terms of sex, age, type of surgery, tumor location, or distance from pylorus to tumor (Table 1).

\section{Pyloric function}

For intervention patients, in regard to decrease in pyloric function, there were a few patients who suffered symptoms of reflux, diarrhea, etc. However, none of the symptoms lasted longer than 6 months during our regular OPC follow-up. In UGIS, done 3 months postoperatively, there were significantly less signs of gastroesophageal reflux in the intervention group compared to the control group ( $0.0 \%$ vs. $10.8 \%, \mathrm{P}=0.021)$. However, for postoperative EGD, done 12 months postoperatively, there was no significant difference between the two groups (Table 2).

\section{Nutritional status}

There was no difference in nutritional status in terms of body weight change and serum albumin levels between the intervention and control group (Table 3).

Table 1. Patient demographics

\begin{tabular}{lccc}
\hline Characteristics & $\begin{array}{c}\text { Control group } \\
(\mathrm{n}=102)\end{array}$ & $\begin{array}{c}\text { Intervention } \\
\text { group }(\mathrm{n}=51)\end{array}$ & P-value \\
\hline Sex & 59 & 30 & 0.908 \\
Male & 43 & 21 & \\
Female & $59.40 \pm 12.22$ & $58.63 \pm 11.77$ & 0.709 \\
Age, mean \pm SD (yr) & & & 0.614 \\
Surgery & 3 & 1 & \\
PPG & 76 & 41 & \\
LAPPG/RAPPG & 23 & 9 & 0.171 \\
LPPG/RPPG & & & \\
Tumor location & 10 & 1 & \\
High body & 30 & 9 & \\
Mid body & 35 & 31 & \\
Low body & 27 & 10 & \\
Antrum & & & \\
Distance from pylorus & & 28 & \\
to tumor (cm) & & 20 & \\
4-9 & 73 & 3 & \\
10-14 & 27 & & \\
15-20 & 2 & & \\
\hline
\end{tabular}

$\mathrm{SD}$, standard deviation; PPG, pylorus preserving gastrectomy; LAPPG, laparoscopic assisted PPG; RAPPG, robot assisted PPG; LPPG, laparoscopic PPG; RPPG, robotic PPG. 


\section{Intervention period}

Most patients presented symptoms of DGE during their first postoperative month (early onset group: 45/51, 88.2\%). There were three patients with symptoms at 1-6 months postoperatively (intermediate onset group: 3/51, 5.9\%) and there were three patients with first signs of DGE as late as greater than 6 months postoperatively (late onset group: $3 / 51,5.9 \%$ ). In the early onset group, balloon only intervention was required in $46.7 \%(21 / 45)$ of cases and $54.3 \%$ $(24 / 45)$ required stent insertion. In the intermediate onset and late onset group, balloon only intervention was required in $66.7 \%(2 / 3)$ of cases and 33.3\% (1/3) required stent insertion (Fig. 2).

\section{Intervention success rate}

Regardless of intervention period, 20 out of 48 patients (41.7\%) were successfully discharged after one balloon intervention. For the remaining 28 patients, a second balloon dilatation was attempted in 11 patients (success rate 45.5\%: 5/11). For the other 17 patients, stent insertion was done and all stent insertion patients were successfully discharged after initial balloon (success rate 100.0\%: 17/17). The six patients who failed their second balloon dilatation eventually required stent insertion. There were no post-intervention related complications.

\section{Mean hospital stay and recurrence of DGE}

The mean hospital stay for single balloon intervention was $7.5 \pm$ 5.1 days, the shortest in our study. When two interventions were required, the mean hospital stay for two balloon dilatations was $19.2 \pm 7.6$ days, whereas the mean hospital stay for one balloon dilatation followed by stent insertion was $16.7 \pm 5.9$ days (Table 4 ).

Table 3. Postoperative nutritional status

\begin{tabular}{lccc}
\hline Characteristics & $\begin{array}{c}\text { Control group } \\
(n=79)\end{array}$ & $\begin{array}{c}\text { Intervention } \\
\text { group }(n=51)\end{array}$ & P-value \\
\hline $\begin{array}{l}\text { Body weight change }(\mathrm{kg}) \\
\quad\end{array}$ & & \\
$\quad$ Postoperative 6 months & $-3.35 \pm 3.96$ & $-3.05 \pm 4.19$ & 0.407 \\
$\quad$ Postoperative 12 months & $-3.41 \pm 4.42$ & $-3.27 \pm 4.54$ & 0.172 \\
Serum albumin change $(\mathrm{g} / \mathrm{dL})$ & & & \\
$\quad$ Postoperative 6 months & $4.28 \pm 0.23$ & $4.25 \pm 0.30$ & 0.465 \\
Postoperative 12 months & $4.32 \pm 0.22$ & $4.28 \pm 0.34$ & 0.730 \\
\hline
\end{tabular}

Values are presented as mean \pm standard deviation.

Table 2. Postoperative pyloric function evaluation by UGIS and EGD

\begin{tabular}{|c|c|c|c|}
\hline Characteristics & Control group $(n=102)$ & Intervention group $(n=51)$ & P-value \\
\hline \multicolumn{4}{|l|}{ UGIS } \\
\hline No specific finding & $51(50.0)$ & $34(66.7)$ & 0.051 \\
\hline Remnant food & $26(25.5)$ & $10(19.6)$ & 0.422 \\
\hline Signs of gastroesophageal reflux (e.g., contrast reflux) & $11(10.8)$ & 0 & $0.021^{\text {a) }}$ \\
\hline Pylorus abnormality (e.g., pyloric spasm, narrowing) & $14(13.7)$ & $7(13.7)$ & 0.872 \\
\hline \multicolumn{4}{|l|}{ EGD } \\
\hline No specific finding & $57(55.9)$ & $32(62.7)$ & 0.421 \\
\hline Remnant food & $28(27.5)$ & $9(17.6)$ & 0.184 \\
\hline Signs of gastroesophageal reflux (e.g., mucosal break, ulcer) & $16(15.7)$ & $10(19.6)$ & 0.546 \\
\hline Pylorus abnormality (e.g., pyloric stenosis) & $1(1.0)$ & 0 & 0.481 \\
\hline
\end{tabular}

UGIS was done at 3 months and EGD was done at 12 months postoperatively.

UGIS, upper gastrointestinal series; EGD, esophagogastroduodenoscopy.

a) Statistically significant, $\mathrm{P}<0.05$

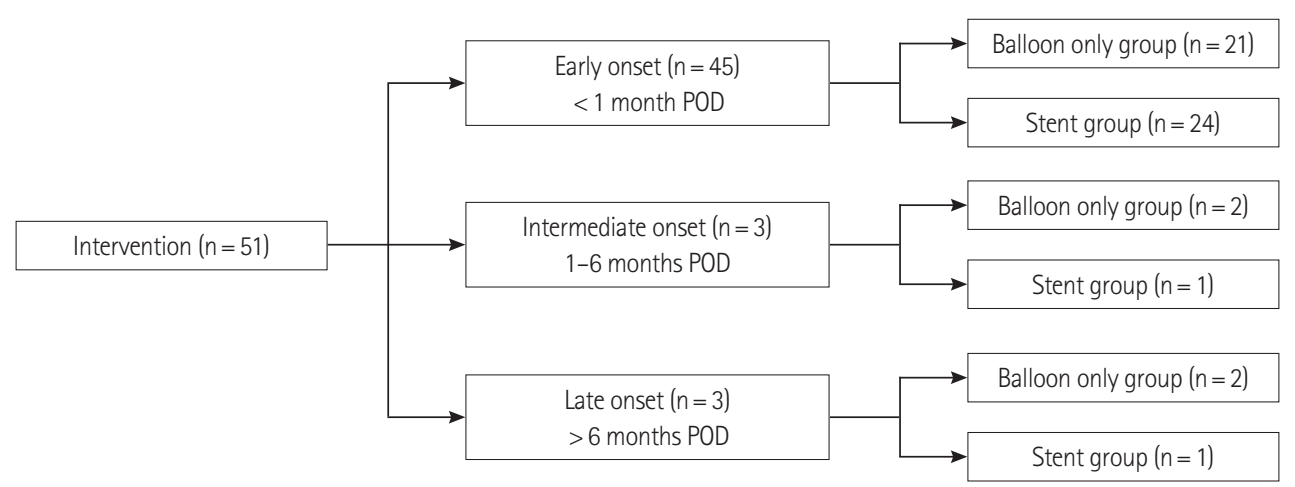

Fig. 2. Intervention according to postoperative day (POD). 
Table 4. Mean hospital stay according to treatment plan

\begin{tabular}{lc}
\hline Intervention type & Hospital stay, mean \pm SD (day) \\
\hline Balloon $\times 1(n=20)$ & $7.5 \pm 5.1$ \\
Balloon $\times 2(n=5)$ & $19.2 \pm 7.6$ \\
Balloon $\times 1 \rightarrow$ stent $\times 1(n=17)$ & $16.7 \pm 5.9$ \\
Balloon $\times 2 \rightarrow$ stent $\times 1(n=3)$ & $18.3 \pm 6.6$ \\
Balloon $\times 2 \rightarrow$ stent $\times 2(n=2)$ & $27.5 \pm 0.5$ \\
Balloon $\times 3 \rightarrow$ stent $\times 1(n=1)$ & $28.0 \pm 0.0$ \\
Stent $\times 1(n=3)$ & $16.0 \pm 2.2$ \\
\hline
\end{tabular}

$\mathrm{SD}$, standard deviation.

The recurrence of DGE for balloon only intervention was significantly higher compared to patients who had stent insertion as part of their treatment (12.0\% vs. $3.8 \%, \mathrm{P}=0.041$ ) (Table 5).

\section{DISCUSSION}

PPG is a function-preserving gastrectomy with comparable oncological outcomes when compared to DG [3-12]. However, postoperative pyloric spasm may occur which can cause DGE. Radiological intervention is an effective treatment of choice for DGE. However, all methods of radiological treatment involve mechanical intervention of the pylorus, which in turn may lead to physical deformities of the pylorus and a decrease in pyloric function. This may result in increased rates of dumping syndrome, gastroesophageal reflux, and, due to the decrease in functionality, may lead to decreased nutritional states compared to the control group [5].

When comparison of patient demographics was done, there was no significant difference between the intervention group and the control group, including tumor location and the length from the pylorus. It has been shown that patients with a short antral cuff length are more susceptible to developing DGE [17]. The fact that there was no difference between the intervention and control group suggests that the intervention group was not more susceptible to developing DGE which allows for a better comparison between the two groups, especially in terms of pyloric function.

The first parameter used to determine pyloric function was patients' symptoms. Precise questions on whether symptoms of dumping syndrome (nausea or bloating) developed were asked of all patients during OPC follow-up. Subjectively, even though there were patients who complained of mild nausea, bloating, gastroesophageal reflux or diarrhea, during the long-term follow-up of patients, there was no long-lasting discomfort for all patients in the intervention or control group. If patients suffered from symptoms suggesting dumping syndrome post-intervention, we can infer that the intervention may have caused a decrease in pyloric function. On the other hand, if patients suffered from increased symp-
Table 5. Recurrence rate according to intervention type

\begin{tabular}{lccc}
\hline Characteristics & $\begin{array}{c}\text { Balloon intervention } \\
\text { only }(n=25)\end{array}$ & $\begin{array}{c}\text { Stent insertion } \\
(n=26)\end{array}$ & P-value \\
\hline Recurrence rate, No. $(\%)$ & $3(12.0)$ & $1(3.8)$ & $0.041^{\text {a) }}$ \\
\hline
\end{tabular}

a) Statistically significant, $\mathrm{P}<0.05$.

toms of gastroesophageal reflux disease, we were able to infer that the patient may have DGE which helped us determine if the patient required intervention. However, due to the retrospective nature of this study questionnaire for gastroesophageal reflux or dumping syndrome was not available, which would have provided a more accurate analysis.

The second parameter determining pyloric function was postoperative UGIS findings, carried out 3 months postoperatively. This data provided objective findings about pyloric function during the relatively early postoperative follow-up period. There were no significant differences between "remnant food" $(\mathrm{P}=$ $0.422)$ and "pylorus abnormality" $(\mathrm{P}=0.872)$. However, there was a significant decrease $(\mathrm{P}=0.021)$ in signs of reflux after intervention. This decrease in gastroesophageal reflux may be due to a decrease in pyloric function shortly after intervention.

However, when the third parameter to determine pyloric function, EGD findings compared 12 months postoperatively, there were no significant differences along all four parameters. This suggests that even if there might have been a short-term decrease in pyloric function due to the intervention, in the relatively longterm, there is no lasting damage to the pylorus.

Although a crude method, our study used changes in body weight and serum albumin levels to determine the patients' nutritional status. There have been studies showing that 6 months postoperatively, there was a significant decrease in body weight and serum albumin levels for DG patients compared to PPG [5]. If mechanical intervention led to a decrease in pyloric function for PPG patients, we can presume that the patients will show findings similar to DG patients. However, in our study there was no difference in nutritional status between the intervention and control group, suggesting that there was no decrease in pylorus function after intervention.

DGE was found to occur in the early stages of postoperative care in our study, more specifically, during the first postoperative month (45/51, 88.2\%). However, our data also showed that there were cases where DGE occurred as late as 304 days postoperatively. Even in such delayed cases of DGE, our center used balloon dilatation as the primary choice of treatment, and stent insertion was used for patients' refractory to balloon dilatation.

Although there were only three patients in each of the time periods between the intermediate onset group (1-6 months) and late 
onset group (greater than 6 months), the interventional success rate for balloon only intervention for these time periods was $66.7 \%$ $(2 / 3)$. Even though the number of patients at this period was too few to conclude that balloon intervention could be used as a primary choice for even delayed events of DGE, our center has shown to have success by doing so.

In our study, 25 patients (49.0\%) were treated with balloon only intervention. In other words, around half (51.0\%) of the patients suffering from DGE required retrievable stent insertion as part of their treatment. This means that it is vital to understand which patients may require stent insertion and if so, when to do it.

There were three patients who underwent initial stent insertion. These patients all had severe pyloric stenosis compared to the other patients, and they were all discharged after just one intervention. The remaining 48 patients all underwent balloon dilatation as their primary choice of treatment and 20 out of 48 patients (41.7\%) were successfully discharged after just one balloon intervention. For the remaining 28 patients with persisting symptoms related to DGE, if balloon dilatation was done, the success rate was $45.5 \%$ (5/11), whereas if stent insertion was done, the success rate was $100.0 \%(17 / 17)$. Furthermore, for the remaining six patients, even if further balloon dilatation was done after two balloon dilatations, they all underwent stent insertion eventually.

This high success rate for retrievable stent insertion in treating DGE can be seen in the patients' mean hospital stay. Even if a single balloon dilatation resulted in the shortest hospital stay of $7.5 \pm$ 5.1 days, for two balloon dilatations, hospital stay increased dramatically to $19.2 \pm 7.6$ days. In contrast, the mean hospital stay for one balloon dilatation followed by stent insertion was $16.7 \pm 5.9$ days. Interestingly, in our study, the mean hospital stay for patients who underwent balloon dilatation two times followed by stent insertion was $18.3 \pm 6.6$ days; shorter than just two balloon dilatations and comparable to just a single stent insertion, where the mean hospital stay was $16.0 \pm 2.2$ days. In terms of recurrence of DGE, patients who underwent stent insertion had a significantly lower recurrence of DGE compared to patients who only underwent balloon intervention ( $3.8 \%$ vs. $12.0 \%, \mathrm{P}=0.041)$. This suggests that even though stent insertion provides long-term relief of DGE symptoms, as mentioned above, it does not cause a decrease in pyloric function.

This study may have several limitations. One main limitation is that due to the retrospective nature, there could be a possibility of selection bias and follow-up of short- and long-term pyloric function was done on two separate exams of UGIS and EGD. The follow-up of symptoms during OPC follow-up was subjective and an accurate analysis of symptoms was limited. The sample size was not large and due to the limited number of patients, accurately de- termining the recurrence of DGE of each intervention may be limited. However, it does show a trend that suggests that stent insertion may be more beneficial in the end. To determine with more certainty the recurrence of DGE, a larger study period involving more patients may be required.

In conclusion, even though radiologic intervention involving the pylorus may decrease its short-term function, there were no differences in its long-term function, and balloon intervention can be used as the first treatment of choice for patients with early until late onset DGE. However, if a second intervention is required subsequent retrievable stent insertion is suggested.

\section{CONFLICT OF INTEREST}

No potential conflict of interest relevant to this article was reported.

\section{ACKNOWLEDGMENTS}

This work was supported by the SNUH Research Fund (grant No. 04-2020-3110).

\section{REFERENCES}

1. Kong SH, Kim JW, Lee HJ, Kim WH, Lee KU, Yang HK. The safety of the dissection of lymph node stations 5 and 6 in pylorus-preserving gastrectomy. Ann Surg Oncol 2009;16:3252-8.

2. Hiki N, Kaminishi M. Pylorus-preserving gastrectomy in gastric cancer surgery: open and laparoscopic approaches. Langenbecks Arch Surg 2005;390:442-7.

3. Hiki N, Nunobe S, Kubota T, Jiang X. Function-preserving gastrectomy for early gastric cancer. Ann Surg Oncol 2013;20:2683-92.

4. Oh SY, Lee HJ, Yang HK. Pylorus-preserving gastrectomy for gastric cancer. J Gastric Cancer 2016;16:63-71.

5. Suh YS, Han DS, Kong SH, Kwon S, Shin CI, Kim WH, et al. Laparoscopy-assisted pylorus-preserving gastrectomy is better than laparoscopy-assisted distal gastrectomy for middle-third early gastric cancer. Ann Surg 2014;259:485-93.

6. Eom BW, Park B, Yoon HM, Ryu KW, Kim YW. Laparoscopy-assisted pylorus-preserving gastrectomy for early gastric cancer: a retrospective study of long-term functional outcomes and quality of life. World J Gastroenterol 2019;25:5494-504.

7. Hotta T, Taniguchi K, Kobayashi Y, Johata K, Sahara M, Naka T, et al. Postoperative evaluation of pylorus-preserving procedures compared with conventional distal gastrectomy for early gastric cancer. Surg Today 2001;31:774-9.

8. Fujita T. Outcome of pylorus-preserving gastrectomy for early gas- 
tric cancer (Br J Surg 2008; 95: 1131-1135). Br J Surg 2008;95:1429.

9. Park DJ, Lee HJ, Jung HC, Kim WH, Lee KU, Yang HK. Clinical outcome of pylorus-preserving gastrectomy in gastric cancer in comparison with conventional distal gastrectomy with Billroth I anastomosis. World J Surg 2008;32:1029-36.

10. Hiki N, Sano T, Fukunaga T, Ohyama S, Tokunaga M, Yamaguchi T. Survival benefit of pylorus-preserving gastrectomy in early gastric cancer. J Am Coll Surg 2009;209:297-301.

11. Kim HH, Han SU, Kim MC, Hyung WJ, Kim W, Lee HJ, et al. Long-term results of laparoscopic gastrectomy for gastric cancer: a large-scale case-control and case-matched Korean multicenter study. J Clin Oncol 2014;32:627-33.

12. Han DS, Suh YS, Ahn HS, Kong SH, Lee HJ, Kim WH, et al. Comparison of surgical outcomes of robot-assisted and laparoscopy-assisted pylorus-preserving gastrectomy for gastric cancer: a propensity score matching analysis. Ann Surg Oncol 2015;22:2323-8.

13. Kaji S, Makuuchi R, Irino T, Tanizawa Y, Bando E, Kawamura T, et al. Preventive effect on delayed gastric emptying of preserving the infra-pyloric vein in laparoscopic pylorus-preserving gastrectomy for early gastric cancer. Surg Endosc 2020;34:3853-60.

14. Imada T, Rino Y, Takahashi M, Hatori S, Tanaka J, Shiozawa M, et al. Gastric emptying after pylorus-preserving gastrectomy in comparison with conventional subtotal gastrectomy for early gastric carcinoma. Surg Today 1998;28:135-8.

15. Isozaki H, Okajima K, Momura E, Ichinona T, Fujii K, Izumi N, et al. Postoperative evaluation of pylorus-preserving gastrectomy for early gastric cancer. Br J Surg 1996;83:266-9.

16. Imada T, Rino Y, Takahashi M, Suzuki M, Tanaka J, Shiozawa M, et al. Postoperative functional evaluation of pylorus-preserving gastrectomy for early gastric cancer compared with conventional dis- tal gastrectomy. Surgery 1998;123:165-70.

17. Nakane Y, Michiura T, Inoue K, Sato M, Nakai K, Yamamichi K. Length of the antral segment in pylorus-preserving gastrectomy. $\mathrm{Br}$ J Surg 2002;89:220-4.

18. Nunobe S, Sasako M, Saka M, Fukagawa T, Katai H, Sano T. Symptom evaluation of long-term postoperative outcomes after pylorus-preserving gastrectomy for early gastric cancer. Gastric Cancer 2007;10:167-72.

19. Morita S, Sasako M, Saka M, Fukagawa T, Sano T, Katai H. Correlation between the length of the pyloric cuff and postoperative evaluation after pylorus-preserving gastrectomy. Gastric Cancer 2010;13:109-16.

20. Jiang X, Hiki N, Nunobe S, Fukunaga T, Kumagai K, Nohara K, et al. Postoperative outcomes and complications after laparoscopy-assisted pylorus-preserving gastrectomy for early gastric cancer. Ann Surg 2011;253:928-33.

21. Hagiwara A, Sonoyama Y, Togawa T, Yamasaki J, Sakakura C, Yamagishi $\mathrm{H}$. Combined use of electrosurgical incisions and balloon dilatation for the treatment of refractory postoperative pyloric stenosis. Gastrointest Endosc 2001;53:504-8.

22. Kim JH, Song HY, Park SW, Yoon CJ, Shin JH, Yook JH, et al. Early symptomatic strictures after gastric surgery: palliation with balloon dilation and stent placement. J Vasc Interv Radiol 2008;19:565-70.

23. Kim JH, Shin JH, Song HY. Benign strictures of the esophagus and gastric outlet: interventional management. Korean J Radiol 2010; 11:497-506

24. Bae JS, Kim SH, Shin CI, Joo I, Yoon JH, Lee HJ, et al. Efficacy of gastric balloon dilatation and/or retrievable stent insertion for pyloric spasms after pylorus-preserving gastrectomy: retrospective analysis. PLoS One 2015;10:e144470. 